\title{
Activity-based nutrition management model for healthcare using similar group analysis
}

\author{
Kyungyong Chung ${ }^{\mathrm{a}}$ and Jonghun Kim ${ }^{\mathrm{b}, *}$ \\ ${ }^{a}$ Division of Computer Science and Engineering, Kyonggi University, Suwon-si 16227, Korea \\ ${ }^{\mathrm{b}}$ Department of Software Convergence Engineering, Inha University, Michuhol-gu 22212, Korea
}

Received 11 January 2019

Accepted 10 April 2019

\begin{abstract}
.
BACKGROUND: Due to environmental factors such as nutrient intake imbalance, lack of exercise, and increased stress, it is necessary to control nutrition in order to prevent diseases and provide treatment in terms of healthcare.

OBJECTIVE: This study proposes the activity-based nutrition management model with the use of the cluster analysis of similar group for healthcare.

METHODS: The proposed method is to conduct the cluster analysis of similar group for nutrition management and to develop the real-time activity information based nutrition management algorithm with the use of big data in order to improve the quality of healthcare management service. It is to re-process an existing nutrition database and add voice recognition function in line with the service so as to improve convenience of intake-food inputs. In addition, the Bluetooth Low Energy (BLE) communication based standard collection of bio signals occurring in real-time is developed. This study also proposes the method of improving an existing algorithm of drawing a daily recommended allowance with the use of real-time activity information, and the proposed service provides the essential information of nutrition management with the use of public big data.

RESULTS: To verify the developed technology and service model and its effectiveness, the nutrition management service system is designed and developed with human interface.

CONCLUSIONS: The developed health model helps to solve the obesity problem, save medical costs, and address the issue of national health.
\end{abstract}

Keywords: Big data, algorithm, ICT, nutrition management, healthcare service

\section{Introduction}

Ageing population and westernized eating habits lead to continuous rises in chronic diseases, which cause an increase in the social burden of medical cost. In Korea, the medical cost for chronic diseases reached 19 trillion Won, and increases $9.2 \%$ annually on average. The prevalence rate of male adults' obesity (the rate of those with BMI 25 or more), which is a cause of chronic diseases, is on the constant rise. Male adults' obesity prevalence rate calculated in 2019 was $42.3 \%$, which is on the constant increase [1]. Such an increase is caused by living habits, such as genetic factors, stress, lack of physical activity, excess drinking, and smoking. Dietary factors including fat, sugar, and sodium intakes are highly

\footnotetext{
${ }^{*}$ Corresponding author: Jonghun Kim, Department of Software Convergence Engineering, Inha University, 100 Inha-ro, Michuhol-gu 22212, Incheon, Korea. E-mail: ddcome11@ daum.net.
} 
related to obesity. As such, obesity is one of the critical causes of chronic diseases, and it is attributable to nutrient intake imbalance. In terms of nutrition intake of each age group, young adults have more intake of nutrients related to obesity, and the intake trend is on the rise [2]. When young adults begins to have physical ageing and reducing muscles, they can have obesity and they can easily be exposed to many different diseases due to their abdominal obesity. As their obesity remains long, it can develop into chronic diseases. As the preemptive measures to prevent aged persons' chronic diseases, nutrition management of young adults is of very importance. Young adults are the main group of economic activity so that they face environmental restrictions, such as space, time, and economy, in order for their nutrition management. For this reason, by utilizing information communication technology, it is necessary to provide them with the activity- based nutrition management service which is aimed at inducing young obese patients to have a desirable dietary habit, to eat balanced nutrients, and keep healthy weight. An existing nutrition management service is mainly based on offline service. The information system based service fails to be activated because of the absence of Information and Communication Technology (ICT) based technology use and inaccuracy of nutrition management algorithm. Since such system and service are developed on the basis of suppliers, users' satisfaction is found to be low. With the constant development of ICT, the field of healthcare has faced paradigm change [3]. The convergence of ICT and an industry makes it possible to implement healthcare regardless of environments. For this reason, the health service expands to wellness based prevention or healthcare beyond treatment [4-7]. Nevertheless, in the nutrition management area, the ICT based technology for nutrition management is not so much developed and applied as it is commercialized, and the algorithm of creating and offering information has low accuracy. In addition, a demander based service model needs to be developed. The nutrition management system for obesity, which emerges as a social issue, is introduced at home and abroad, and its clinical effect is announced. There have been a lot of investments and studies on the development of devices and solutions which can be used regardless of time and space. In the healthcare area, the world sees the change of dietary life, over-nutrition, and lack of exercise. As a result, cardiovascular diseases, dyslipidemia, arteriosclerosis, and heart disease occurred more often [8].

In this study, an activity-based nutrition management system is developed to improve online and offline nutrition management service fundamentally, and its service effectiveness is verified. In order for the system to be applied to users and service in reality, an intake amount is converted into the unit of a paper cup and a nutrition analysis database is reestablished. The established database is applied to establish the model of the voice recognition system, and to build the voice recognition engine which is used to recognize a variety of food and an amount of intake as continuous voice. For the mobile healthcare service, the ICT based method of transmitting and changing the standard data is developed to collect real-time bio data. In addition, the algorithm of calculating a calorie of daily intake with the use of activity information is developed, and the nutrition management system provides the information created after the process of public health big data. The requirements of members of nutrition management service are applied to draw a commercial service model which is applied to system design for development. The developed system is verified in terms of function and effectiveness.

\section{Activity-based nutrition management technology}

\subsection{Establishment of nutrition analysis database for nutrition management service}

Three thousand six hundred and fifty-seven food products and 1,821 food types of Food Product and Food Nutrients Database of Korean Nutrition Society [9], 203 food products and 1,819 food types are reprocessed by Dietetic Institute [10] in the order of the highest consumption and highest frequency food 


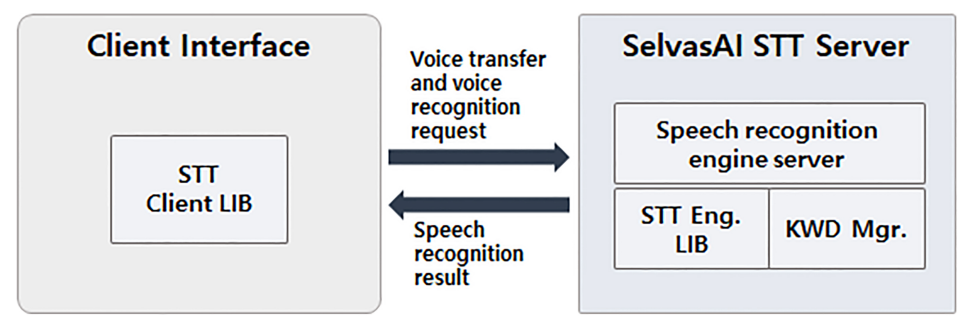

Fig. 1. Voice recognition process.

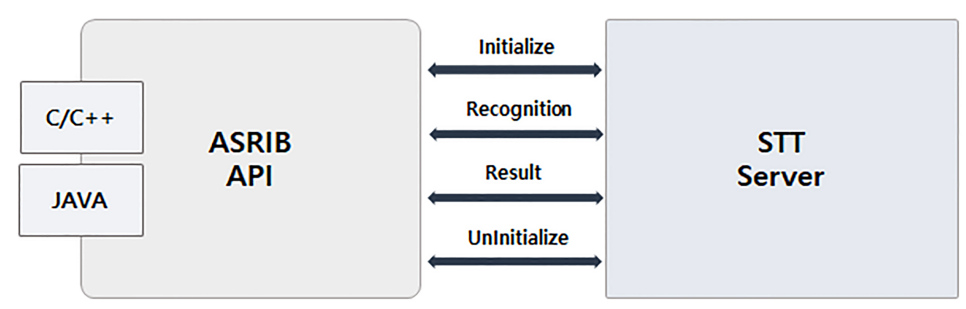

Fig. 2. Recognition server interface.

types of adult Koreans. As a unit of a paper cup, there are 0.25 ( $1 / 4$ cup), 0.5 ( $1 / 2$ cup), 0.75 (3/4 cup), and 1 ( 1 cup) types on the basis of the paper cup of $180 \mathrm{ml}$. The food product and food amounts of the Korean Nutrition Society database are converted into the paper cup amounts of portion sizes (g) so as to establish the nutrition analysis database for nutrition management service. The items with large errors of weight $(\mathrm{g})$ and volume $(\mathrm{ml})$ are actually measured. In terms of the items that include soup and solid ingredients, the paper cup unit conversion formula for the items is developed and applied.

\subsection{Automatic food product recognition technology using voice recognition}

Voice recognition is the computer technology of analyzing voice language and converting data into characters. A voice is the means of communication naturally used in everyday life and does not need any expensive device in voice input and transmission. Voice recognition technology is divided into speakerdependent technology and speaker-independent technology. The speaker-dependent technology is able to recognize a specific speaker depending on recognizable persons. Voice recognition technology is able to recognize voice according to a pronunciation type and the number of vocabularies. In terms of recognition based on pronunciation types, there are the keyword detection methods able to detect and recognize isolated words, connected words, continuous sentences, and conversational continuous sentences, and specific words. In terms of recognition based on the number of vocabularies, there are smalls size (ten to hundreds of words) recognition technology and large size (tens of thousands of words) recognition technology. In case of the voice recognition with complex structure, the final recognition result is drawn through the language model based language processing.

Figure 1 illustrates the voice recognition procedure using STT server of SelvasAI [11]. For the voice recognition to be applied to nutrition management system, the voice recognition system of SelvasAI is used. For the recognition of unspecified speakers' diverse food name and utterance sentence, the speakerindependent and large size continuous speech recognition technology is applied. The nutrition database established in the system development process is applied to newly refine the sound model and language model suitable for nutrition management service and optimize the DNN based voice recognition engine. 
Depending on service models, the sound model in diverse environments is established. The model established on the basis of nutrition analysis database is applied to continuous speech voice recognition system for Korean language so as to develop the voice recognition function fitting the activity-based nutrition management system.

As shown in Fig. 2, recognition server interface is developed and established to receive a recognition result in real time regardless of a type of voice information transmission. When the request of voice recognition is given to Server with the use of the functions defined in ASRLIB API, its recognition result is sent to a client program. With the uses of Initialize and UnInitialize functions, the connection information (IP, Port) used for API are set up, and released. If additional connection information is needed, it is possible to set up the information. Recognition and Result functions are used to send voice information, and a recognition result is sent back after Server recognition is completed.

\subsection{Ambient device interaction technology using bio-signals}

In a telecommunication environment, ambient intelligence technology has been developed. In the circumstance, smart devices using wearable sensors are used to analyze bio data in everyday life. A lot of investments and studies have been made on the customized healthcare service which makes it possible to monitor one's own condition through telecommunication [12-15]. As an example, there are smart devices capable of measuring activity or heart rate, such as glasses, bands, shoes, and belts [16-19]. Smart shoes and bands use IMU sensor including accelerator and gyroscope in order to measure bio data such as a user's movement, motion, and heart rate, and record position data in everyday life through GPS sensor. Based on these collected data, personal activity pattern and heart rate pattern are analyzed so as to provide the customized healthcare service [9].

For an ambient intelligence based healthcare, it is possible to utilize ISO/IEEE 11073-20601 protocol in which the data exchange between a smart device for measuring personal bio data and a gateway is defined. IEEE 11073-20601 protocol consists of Domain Information Model, Service Model, and Communication Model [20]. Bluetooth Low Energy (BLE), which is a typical communication protocol of bio signal measurement devices and smartphones, is also called Bluetooth Smart. For lightweight classic Bluetooth version, a part of Bluetooth 4.0 was announced. BLE provides Bluetooth wireless connectivity for an inexpensive small battery based device that needs several months to several years of battery lifespan. BLE technology contributes to the scalability of the healthcare service, since it is applied to watches, Proximity tags, sports and appropriate fitness sensors, healthcare sensors and remote controllers. In the activity-based nutrition management system, InBodyBAND2 of Inbody, PWB-100, 200, and 250 of Parton, Ribbon Device of Uracle, and UA-651BLE of A\&D interact with BLE over IEEE 11073 protocol.

\section{Similar group analysis and activity-based nutrition management}

\subsection{Development of nutrition management algorithm reflecting each stage of activity}

Calorie control based nutrition management is an issue of present-day's people. Most systems propose a target intake calorie or recommend food menu or diet. An advanced nutrition management system implements the daily recommended calorie intake formula which is used offline nutrition management service in a software type and offers nutrition management service. Unlike the offline nutrition management service, the system uses a user's fixed activity information or does not collect information 
Table 1

Definition of physical activity levels

\begin{tabular}{lclc}
\hline \multicolumn{1}{c}{ Category of activity } & \multicolumn{1}{c}{ Low active level } & \multicolumn{1}{c}{ Active level } & \multicolumn{1}{c}{ Very active level } \\
\hline $\begin{array}{l}\text { Level of physical ac- } \\
\text { tivity }\end{array}$ & $1.50(1.40 \sim 1.60)$ & $1.75(1.60 \sim 1.90)$ & $2.00(1.90 \sim 2.20)$ \\
Everyday life & $\begin{array}{l}\text { Static activity of sit- } \\
\text { ting most of the time }\end{array}$ & $\begin{array}{l}\text { Mostly sitting; some standing work, com- } \\
\text { muting, product buying, household work, cise; active leisure activity } \\
\text { and light exercise }\end{array}$ \\
\hline
\end{tabular}

automatically so that it fails to provide a daily recommended intake calorie accurately. A calorie generated in the body for a certain time is referred to as metabolism. As a unit, there are calorie (cal) and kilo calorie (kcal). Humans consume energy for their basic activity even in their sleep in order to keep their body temperature and heart rate. In other words, basic energy is consumed in the body without any movement, which is called basal metabolism. A basic metabolic rate accounts for a great deal of daily consumed energy so that it is of importance. Energy expenditure other than that is mostly made for body movement activities. All body 'activities', such as exercise, singing, and dancing, lead to energy expenditure, which is called activity metabolism. In terms of a degree of obesity, a weight can be evaluated with Body Mass Index (BMI). Of the indexes based on weight and height, BMI is the most widely applied one. It is calculated in the way of dividing one's weight $(\mathrm{kg})$ by the square $\left(\mathrm{m}^{2}\right)$ of its height. A standard weight is calculated in either Broca formula or modified Broca formula. If a standard height is $161 \mathrm{~cm}$ or more, Broca formula is applied; if $160 \mathrm{~cm}$ or less, modified Broca formula is applied. Information on muscle mass and body fat percentage is helpful for nutrition judgment. Such judgment is better than the judgment only with BMI. In terms of a daily recommended intake calorie, a daily energy requirement can be calculated differently depending on general adults, obesity, and low weight. Other nutrition requirements are calculated generally on the basis of the official 'Dietary Reference Intakes for Koreans'. For instance, according to 2015' Dietary Reference Intakes for Koreans, the physical activity of male and female adults aged 20 to 49 was $1.55 \pm 0.19$ and $1.46 \pm 0.17$, respectively, which means 'low active $(1.4 \leqslant \mathrm{PAL}<1.6)$. If one is allowed to select its own physical activity level (low active, active, very active) in its physical information record, in terms of the estimated energy allowance for low activity, women can have 1.12 of PA (Physical Activity) and men 1.11. Accordingly, by applying PA differently depending on a activity level (low active, active, and very active), it is possible to calculate an estimated energy requirement. In case of obesity, an estimated energy requirement can be calculated as shown in the below Eq. (1).

$$
662-9.53 \times \text { Age (years) }+ \text { PA }[15.91 \times \text { Adjusted weight }(\mathrm{kg})+539.6 \times \text { Height }(\mathrm{m})]
$$

For example, if an obese man of $170 \mathrm{~cm}$ in height and $76 \mathrm{~kg}$ in weight aims to reach $72 \mathrm{~kg}$ (BMI 24.9), his estimated energy requirement is $2153 \mathrm{kcal}$. If he takes $2400 \mathrm{kcal}$, he needs to do more physical activity so as to burn (2400-2153) kcal. It is desirable to determine PA on the basis of daily living patterns, rather than occupations. It is possible to refer to the below Table 1, where inactive levels can be treated equally as in basal metabolic rate.

A user of nutrition management app selects a level of physical activity in the Table 1 when information input is displayed on the initial screen of the app. Based on the initially input information, the system provides nutrition management guidelines and a daily recommended intake calorie. The user is able to perform its own nutrition management after receiving the guidelines from the system. If its different activity information depending on situations is not entered accurately and manually, it is hard for the system to provide a daily intake calorie accurately. With the use of the bio signal measurement device interaction technology developed in this study, activity information is received from a variety of 


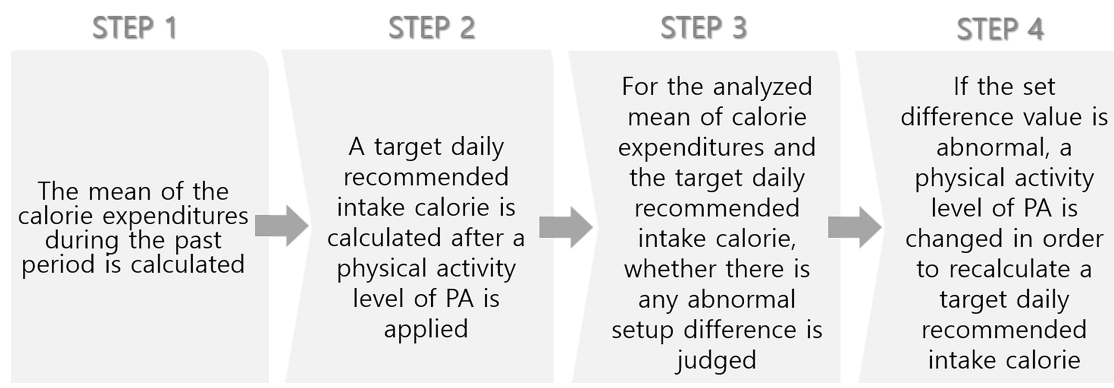

Fig. 3. Nutrition management activity level change process.

wearable devices and is applied to a nutrition management algorithm. The activity information of wearable devices includes walking, stair climbing, running, and other motion information. After three-week activity analysis, a user's average calorie expenditure is calculated. If a user's use period continues, the calorie expenditure information of a similar group is applied so as to draw a user's average calorie expenditure. In the system, an energy requirement is calculated in consideration of a level of physical activity (inactive level, low active level, active level, and very active level). If the already set difference value exceeds positively $(+)$, an activity level goes up. If it is negative (-), the level goes down. If a user's daily energy requirement provided by the system has the difference of $+300 \mathrm{Kcal}$ and more from the activity information (average calorie expenditure) drawn from the app, the system goes up the actual activity level entered by the user (e.g., low active $\rightarrow$ active) when calculating and suggesting the user's calorie requirement. If the difference is $-300 \mathrm{Kcal}$, the system goes down the actual activity level entered by the user (e.g., low active $\rightarrow$ inactive) when calculating and suggesting the user's calorie requirement. If the difference is $\pm 600 \mathrm{kcal}$ and more, it is possible to go up or down two levels. Wearable devices can be used in connection with mobile phones, waist belts, wrist bands, hair bands, glasses, and necklaces. A target daily recommended intake calorie is determined after at least one of weight adjustment target, age, weight, height, sex, and activity level is applied. Weight adjustment target is categorized into weight maintenance, weight reduction, and weight increase. The steps of daily recommended intake calculation using a physical activity level of PA are presented in Fig. 3.

As shown in Fig. 3, the activity-based nutrition management is able to correct a level of physical activity according to real-time activity, to draw a daily recommended intake calorie, and to provide nutrition management information accurately.

\subsection{Similar group analysis algorithm using big data}

The activity-based nutrition management system provides big data analysis information through a user's mobile device app. For the analysis on similar group, National Health Insurance Public Data is used. In other words, the average BMI of particular subjects and general men in similar residential area, the average BMI of general men in all areas, the average BMI of the age group in residential area, the average BMI of the age group in all areas and a particular person's BMI are analyzed. With the use of the National Health Insurance Public Data, the average BMI of general men similar to particular subjects by income quintiles, the average BMI of general men by activity time, the average BMI of general men by occupations, and a particular person's BMI are displayed in graph on app. In this way, the correlation between subjects and unspecified many persons similar to the subjects is intuitively offered in terms of obesity information. Therefore, the development purpose of the proposed algorithm is to improve the quality of nutrition management service and induce the continuous use of the app. For big data analysis, 
Table 2

Similar group analysis factors and target values for obesity and nutrition management

\begin{tabular}{lll}
\hline \multicolumn{1}{c}{ Category } & \multicolumn{1}{c}{ Factor value } & \multicolumn{1}{c}{ Result value } \\
\hline User BMI & Height, weight & User BMI \\
The mean of general men in residential area & Height, weight, sex, residential area & $\begin{array}{l}\text { The average BMI of all similar } \\
\text { group and user BMI position }\end{array}$ \\
The mean of general men in all areas & Height, weight, sex, all areas & \\
The mean of the age group in residential area & Height, weight, sex, residential area, age & \\
The mean of the age group in all areas & Height, weight, sex, all areas, age & \\
The mean of general men by income quintiles & Height, weight, sex, income quintiles & \\
The mean of general men by activity time & Height, weight, sex, activity time & \\
The mean of general men by occupations & Height, weight, sex, occupation & \\
\hline
\end{tabular}

Table 3

Similar group analysis flow

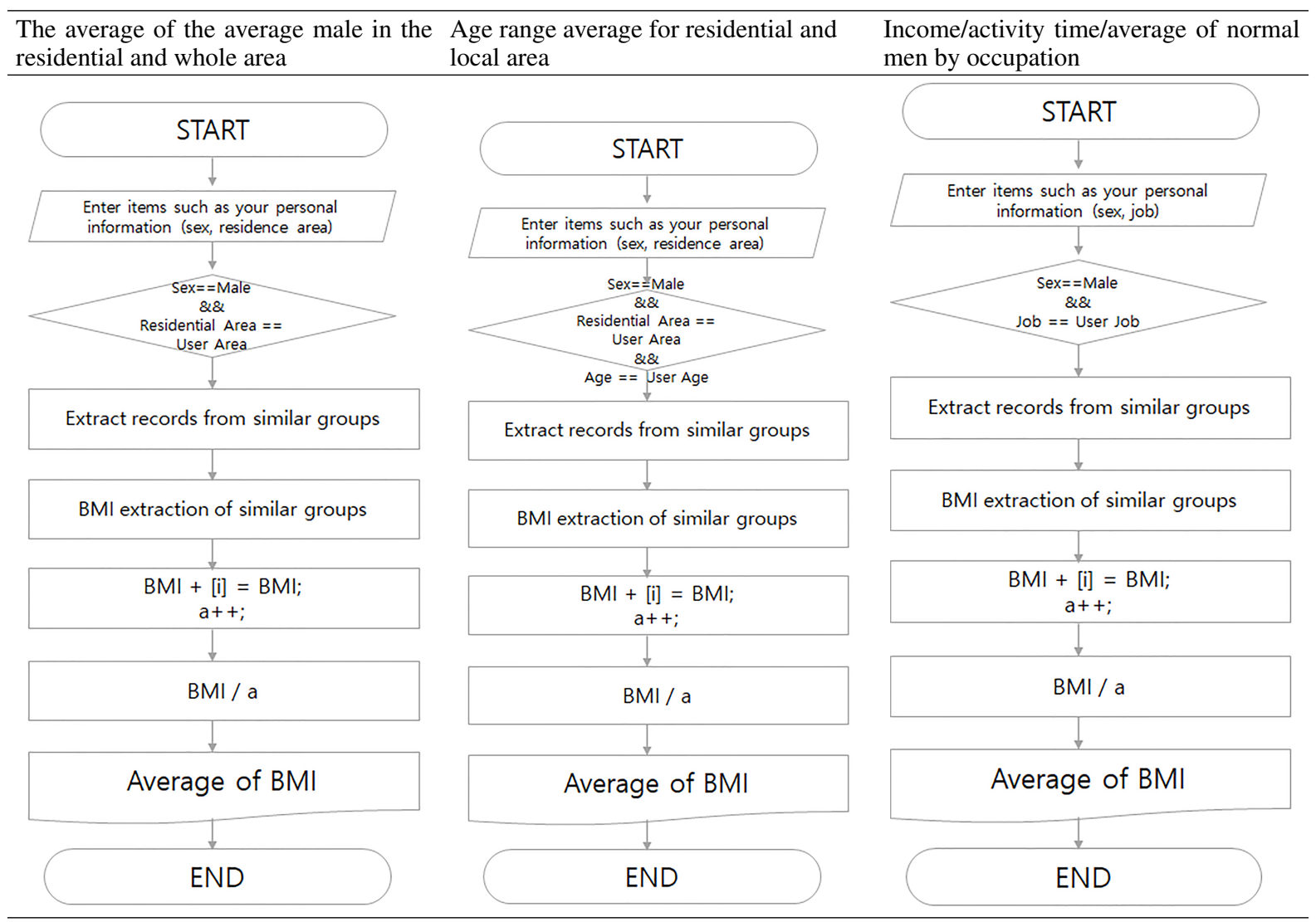

of 34 National Health Insurance raw data of National Health Insurance Corporation, 10,000 items are analyzed. As the analysis result, the factors most related to obesity-height, weight, sex, residential area, and age-are drawn from a similar group. In addition, of 756 National Health and Nutrition Examination Survey items of Korea Centers for Disease Control and Prevention, 8,000 data are analyzed so as to draw the factors related to nutrition management, which are height, weight, sex, income quintiles, activity time, and occupation. Table 2 shows the analysis results according to the drawn factors, and Table 3 presents the system process flow for similar group analysis. 


\section{Development of activity-based nutrition management health model}

\subsection{Activity-based nutrition management health model}

With the extended human life and westernized eating habits, more nutrition management has been needed, and all age groups have been more interested in nutrition management. In this circumstance, it is necessary to provide the activity-based nutrition management health service which makes it possible to manage nutrition easily and continuously in social life and personal life [21-24]. To draw the essential functions for nutrition management, 150 subjects who used or use a nutrition application are selected. They are asked to use three nutrition management service applications for 10 days and more, and to evaluate the importance and performance of each function on the 5-point scale. The service functions that have a significant difference in terms of analysis values and are considered important for nutrition management by general consumers, and work well in nutrition management applications are drawn. Based on the drawn service functions, a level of requirement of nutrition management service functions is analyzed with 400 general consumers. The differences in a level of requirement are analyzed depending on age groups (20-30 years of age group, 40-50 years or more of age group) and whether to have a disease. In the survey of nutrition management service function requirement, the items that a high requirement value in all age groups are categorized into high, middle, and low types, and the items that have a high requirement value are categorized according to whether diseases are included $[25,26]$. Through the meeting of experts including nutrition expert, service expert, clinical medical doctor, exercise expert, and development expert, the basic functions of nutrition management are drawn as shown in Table 4.

Based on the drawn basic functions of nutrition management service, a nutrition management health model is developed. The developed service model uses the interactive voice recognition technology for obese patients so as to take into account their use convenience. Through the activity-based sensing data collection, their activity is accurately analyzed. The quality of the health service is improved in the way of establishing a nutrition database and using a nutrition management algorithm. Figure 4 illustrates the conceptual diagram of the activity-based nutrition management health service model.

\subsection{Activity-based nutrition apps and web systems}

Based on the developed nutrition management analysis database and service model, a nutrition management database with total of tables is developed. In the code system of food type database, a category has two digits, a division 1 digit, and a section three digits. In the code system of food product database, a category has two digits, a division 1 digit, a section three digits, subsection two digits, and blank 1 digit. Through the implemented interaction with bio measurement devices, the developed system collects and analyzes body fat and activity information (step count and calorie expenditure) in interaction with InbodyBand 2 in order to provide the analyzed information to users. Figure 5 shows the screen of device interaction for measuring an activity with the use of InbodyBand2.

The nutrition management system provides such functions as eating record, band interaction, and nutrient evaluation, and side menu is developed in order to search for use history. The web screen is designed for nutritionists to find and analyze members' eating records, menu, nutrients, and calories all at once. In the nutrition management screen, the activity data collected in connection with nutritionists' guidelines, weight management, eating records, and InbodyBand 2 are displayed. These data are analyzed by the system so as to display such information on a daily intake calorie and proper nutrition balance, which are needed for a user's nutrition management. Eating records can be made by a manual way or by 
Table 4

The basic functions of nutrition management information service

\begin{tabular}{|c|c|c|c|}
\hline Function & Category & Details & $\begin{array}{l}\text { Level of } \\
\text { requirement }\end{array}$ \\
\hline \multirow{7}{*}{$\begin{array}{l}\text { Personal } \\
\text { information } \\
\text { input }\end{array}$} & $\begin{array}{l}\text { Basic physical condition } \\
\text { information }\end{array}$ & Personal information input (e.g., sex, age, weight, height) & High \\
\hline & Dietary information & Input of breakfast, lunch, and dinner menu & Middle \\
\hline & & Input of breakfast, lunch, and dinner meal size & Middle \\
\hline & & $\begin{array}{l}\text { Whether to request a manager to add the menu which is not } \\
\text { found in menu search }\end{array}$ & High \\
\hline & & Input of snack intakes & Middle \\
\hline & Goal setting & Target weight loss setting (obesity, dietary habit improvement) & High \\
\hline & & Target daily exercise (e.g., step count, exercise time) setting & High \\
\hline \multirow{10}{*}{$\begin{array}{l}\text { Customized } \\
\text { information } \\
\text { offering }\end{array}$} & Customized basic physical & Offering of BMI based obesity level & High \\
\hline & condition information & $\begin{array}{l}\text { Offering of the information as to whether there is the danger of } \\
\text { high blood pressure }\end{array}$ & Middle \\
\hline & & $\begin{array}{l}\text { Offering of the information as to whether there is the danger of } \\
\text { diabetes }\end{array}$ & Middle \\
\hline & & Offering of daily recommended intake calorie & Middle \\
\hline & Customized dietary & Offering of the calorie for the described meal size & High \\
\hline & information & $\begin{array}{l}\text { Display of the ratio of carbohydrate, protein, and fat for the } \\
\text { described meal size }\end{array}$ & Middle \\
\hline & & Display of the sodium amount for the described meal size & Middle \\
\hline & & Display of the nutrient balance for the described meal size & Middle \\
\hline & & $\begin{array}{l}\text { Offering of essential nutrients after the nutrition analysis on the } \\
\text { described meal }\end{array}$ & High \\
\hline & & $\begin{array}{l}\text { Recommendation of proper food products and food types after } \\
\text { the nutrition analysis on the described meal }\end{array}$ & High \\
\hline \multirow{14}{*}{$\begin{array}{l}\text { General } \\
\text { information } \\
\text { offering }\end{array}$} & Food nutrition & Offering of food recipe & High \\
\hline & & Offering of the latest news about food nutrition & Middle \\
\hline & & Offering of daily recommended menu & Middle \\
\hline & & Offering of the recommended menu for each disease & High \\
\hline & & Offering of the nutrient contents of each food type & High \\
\hline & & Offering of the nutrient contents of each food product & High \\
\hline & & Offering of the one-time provision size of each food product & High \\
\hline & Symptoms and diseases & Offering of obesity management information & Middle \\
\hline & & Offering of high blood pressure information & High \\
\hline & & Offering of diabetes information & High \\
\hline & & Offering of the latest news about diseases & Middle \\
\hline & Physical activity and & Offering of the latest news about physical activity and exercise & Middle \\
\hline & exercise & Offering of the video of each exercise type & High \\
\hline & & Offering of living information & Middle \\
\hline \multirow[t]{3}{*}{ Communication } & Expert & Personal coaching of nutrition expert & Middle \\
\hline & & $\begin{array}{l}\text { FAQ with application developer or administrator in case of app } \\
\text { problem }\end{array}$ & High \\
\hline & Community & Community of app users & Middle \\
\hline \multirow[t]{3}{*}{ Other functions } & Entertainment function & Points saving through attendance check & High \\
\hline & Online shop & Purchase of health and nutrition food products & High \\
\hline & Additional functions & Additional functions in connection with other equipment & Middle \\
\hline
\end{tabular}

the continuous voice based on voice recognition technology. By entering information on food type, food product, and a meal size in such ways, it is possible to improve users' convenience. A manager is able to find and manage service app users' information in the member management of nutrition management web. In terms of food type management function, it is possible to find the classification and code of food type and food product, nutrients of reprocessed database, and other information [27]. By analyzing app users' eating records and nutrients with the use of nutrition management function, a manager is able to provide nutrition guidelines to users directly. As contents, there is information on the guidelines for high 

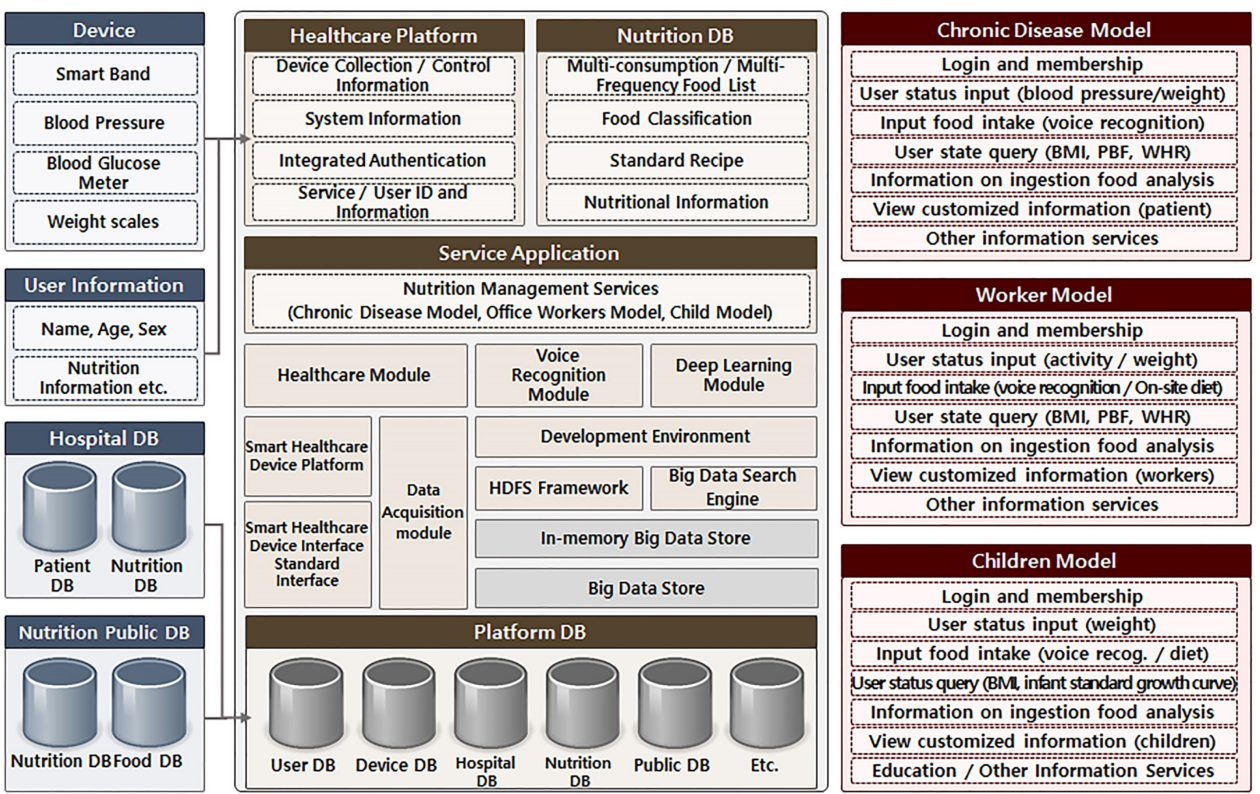

Fig. 4. Activity-based nutrition management health service model.
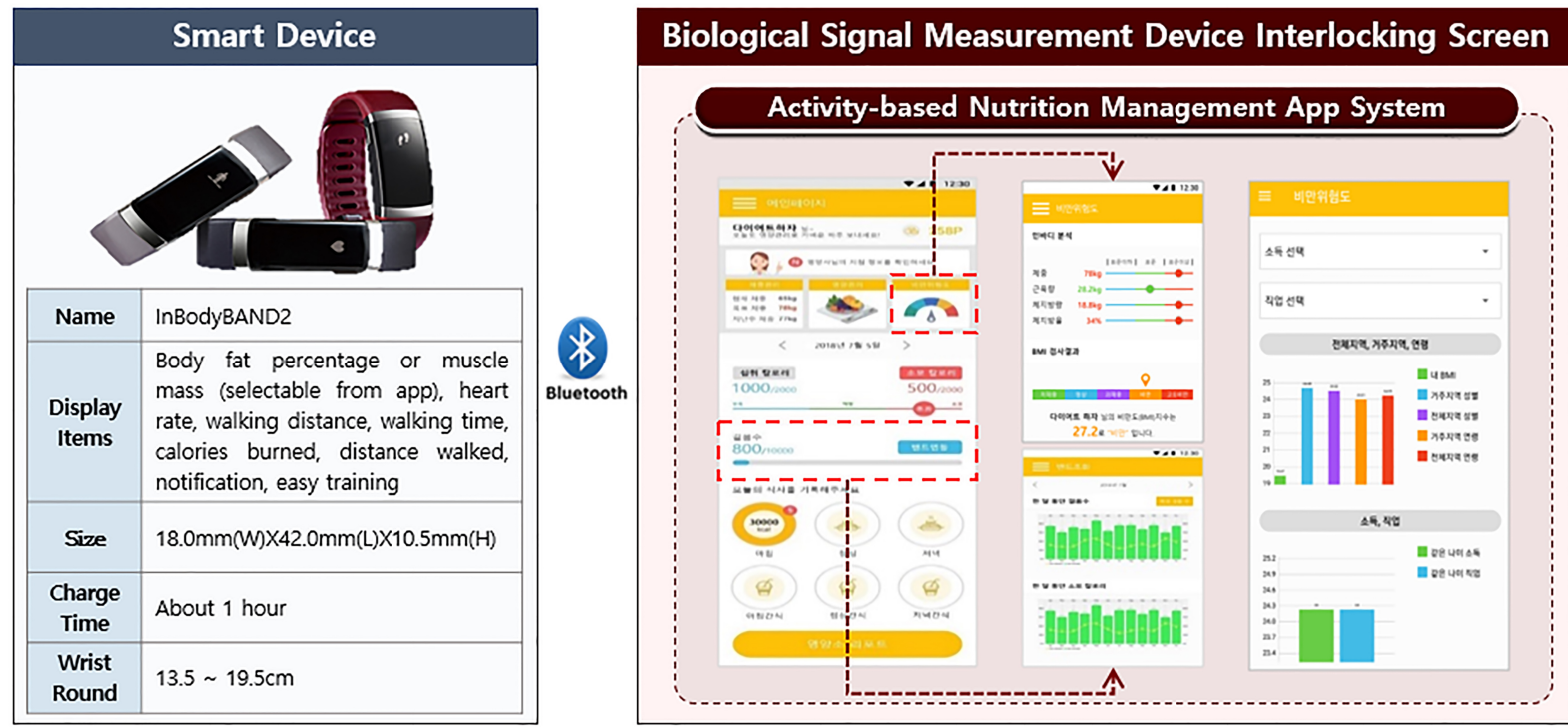

Fig. 5. Band linkage and activity measurement information output view.

sodium, high fat, and weight loss.

\subsection{Performance evaluation}

For the evaluation of the developed system, Institutional Review Board (IRB) approval is obtained from a general university hospital [21]. We provide online nutrition services to 50 people and offline nutrition services to 25 people. The performance of the system is evaluated for three months. Through the 
Table 5

Service performance time differences between online and offline services

\begin{tabular}{ccccc}
\hline Service type & Consultation time & Nutrition judgment & Nutrition consultation & Evaluation and management \\
\hline Online & 15 minutes & Immediately & Immediately & 10 minutes \\
Offline (conventional) & 60 minutes & 30 minutes & 30 minutes & 30 minutes (result sheet) \\
\hline
\end{tabular}

dietary life questionnaire, nutrition evaluation, and satisfaction survey, the effectiveness of the service model and system is analyzed. The comparison of online and offline services are made, and experimental application is implemented in order to verify the logical validity and effectiveness of the health model. According to the evaluation, the functional necessity of the system and the technical satisfaction were high, and the online service was better than an offline service in terms of service convenience, economy, and differentiation. Table 5 shows the service performance time differences between the activity-based nutrition management system and an existing offline nutrition management service. The online service shorten service times more than the offline service in the categories of consultation time, nutrition judgment, nutrition consultation, and evaluation and management.

As the result of the system and service evaluation, the online service of the activity-based nutrition management system has the following advantages:

If nutrition evaluation and consultation are made online, there are no temporal and spatial restrictions. Users are able to find in real time the analyzed calorie and nutrients of what they eat so that they can be aware of daily intake calories and control their calories. In addition, whenever they can have nutrition consultation, they can ask nutritionists questions freely. Through nutrition guidelines, they are able to recognize their dietary habits for weight loss and practice better eating habits in everyday life. In interaction with their smart bands, they are able to receive information on step count and calorie expenditure so that they can get motivation of doing exercise.

\section{Conclusions}

The obesity of young adults, which emerges as a social issue, is caused by living habits which fail to be managed due to environmental problems such as economic problem and time. In terms of the management of living habits important to prevent chronic diseases, obesity management is of significance, and obesity can be prevented and improved by nutrition management. Nevertheless, for the activitybased nutrition management system development, healthcare technology, algorithm, and service model are researched in the academic dimension. Proper food product treatment and nutrient adjustment help to treat diseases and prevent complications, through which it is possible to achieve healthcare. This study redesigned the nutrition analysis database which can be used practically and supports the nutrition management for diseases. For the improvement in users' convenience, continuous voice recognition technology was applied. In interaction with a variety of biometric devices, users' activity was measured depending on their situations. In the system developed in consideration of users' convenience and activity, an existing nutrition management algorithm is improved with the use of real-time activity information. Through the similar group clustering analysis, accurate attention information and measures are provided to users. In the thorough analysis of demands and requirements, human interface was developed so as to verify its effectiveness. In addition, the developed health model helps to solve the obesity problem, save medical cost, and address the issue of national health.

In the future research, it will be necessary to apply the 3D image recognition using status detection to the nutrition management system that draws a lot of public attention, and thereby to develop the 
technology for giving a specialized service to each user. In addition, with the use of the knowledge base based context recognition multi-modal deep learning, it will be required to develop the knowledge processing technology of predicting the risk of obesity and creating diet success or fail information automatically.

\section{Acknowledgments}

This work was supported by the Inha University research grant.

\section{Conflict of interest}

None to report.

\section{References}

[1] Korea Health Insurance Review and Assessment Service [homepage on the Internet]. 2017 [updated 2017; cited 2018 Oct 23]. Available from: http://www.hira.or.kr/eng/main.do.

[2] Korea Centers for Disease Control and Prevention [homepage on the Internet]. 2017 [updated 2018 Oct 7; cited 2019 Nov 12]. Available from: http://www.cdc.go.kr/CDC/eng/.

[3] Lee JY, Lim JY. The prospect of the fourth industrial revolution and home healthcare in super-aged society. Annals of Geriatric Medicine and Research 2017; 21(3): 95-100.

[4] Chomutare T, Fernandez-Luque L, Arsand E, Hartvigsen G. Features of mobile diabetes applications: Review of the literature and analysis of current applications compared against evidencebased guidelines. Journal of Medical Internet Research 2011; 13(3): e65.

[5] Jung H, Chung K. PHR based life health index mobile service using decision support model. Wireless Personal Communications 2016; 86(1): 315-332.

[6] Yoo H, Chung K. PHR based diabetes index service model using life behavior analysis. Wireless Personal Communications 2017; 93(1): 161-174.

[7] Jung H, Chung K. Sequential pattern profiling based bio-detection for smart health service. Cluster Computing 2015; 18(1): 209-219.

[8] Kim J, Jang H, Kim JT, Pan H, Park RC. Big-data based real-time interactive growth management system in wireless communications. Wireless Personal Communications 2018; 105(2): 655-671.

[9] The Korean Nutrition Society [homepage on the Internet]. 2018 [updated 2018; cited 2018 Aug 13]. Available from: http://www.kns.or.kr/English/.

[10] Food Habits Institute [homepage on the Internet]. 2017 [updated 2018; cited 2018 Aug 20]. Available from: http://www. foodcoach.co.kr/.

[11] SELVAS AI [homepage on the Internet]. 2017 [updated 2018; cited 2018 Aug 13]. Available from: https://www.selvasai. $\mathrm{com} / \mathrm{en} /$.

[12] Kim JH, Kim JK, Lee DS, Chung KY. Ontology driven interactive healthcare with wearable sensors. Multimedia Tools and Applications 2014; 71(2): 827-841.

[13] Althenyan Q, Yaseen Q, Jararweh Y, Al-Ayyoub M. Cloud support for large scale e-healthcare systems. Annals of Wireless Communications 2016; 17(9-10): 503-515.

[14] Celdrán AH, Pérez MG, García Clemente FJ, Pérez GM. Preserving patients' privacy in health scenarios through a multi context-aware system. Annals of Wireless Communications 2017; 72(9-10): 577-587.

[15] Kim SH, Chung K. Emergency monitoring service using context motion tracking of chronic disease patients. Cluster Computing 2015; 18(2): 747-759.

[16] Sebbak F, Benhammadi F. Majority-consensus fusion approach for elderly IoT-based healthcare applications. Annals of Wireless Communications 2017; 72(3-4): 157-171.

[17] Haghi M, Thurow K, Stoll R. Wearable devices in medical internet of things: Scientific research and commercially available devices. Journal of Healthcare Informatics Research 2017; 23(1): 4-15.

[18] Chung K, Kim JC, Park RC. Knowledge-based health service considering user convenience using hybrid Wi-Fi P2P. Information Technology and Management 2016; 17(1): 67-80. 
[19] Jung H, Chung K. Life style improvement mobile service for high risk chronic disease based on PHR platform. Cluster Computing 2016; 19(2): 967-977.

[20] ISO [homepage on the Internet]. IEEE 11073-20601: Health informatics-person health device communication, application profile optimized exchange protocol; 2018 [updated 2018; cited 2018 Aug 2]: http://www.iso.org.

[21] Catholic Kwandog University International St. Mary's Hospital [homepage on the Internet]. 2018 [updated 2018; cited 2018 Sep 1]. Available from: http://www.ish.or.kr/eng/.

[22] Kim JC, Chung K. Prediction model of user physical activity using data characteristics-based long short-term memory recurrent neural networks. KSII Transactions on Internet and Information Systems 2019; 13(4): 2060-2077.

[23] Chung K, Yoo H. Blockchain network based topic mining process for cognitive manufacturing. Wireless Personal Communications 2019; 105(2): 583-597.

[24] Kim JC, Chung K. Mining based time-series sleeping pattern analysis for life big-data. Wireless Personal Communications 2019; 105(2): 475-489.

[25] Kim JC, Chung K. Mining health-risk factors using PHR similarity in a hybrid P2P network. Peer-to-Peer Networking and Applications 2018; 11(6): 1278-1287.

[26] Yoo H, Chung K. PHR based diabetes index service model using life behavior analysis. Wireless Personal Communications 2017; 93(1): 161-174.

[27] Kim J, Chung K. Depression index service using knowledge based crowdsourcing in smart health. Wireless Personal Communication 2017; 93(1): 255-268. 